\title{
Segmental pulmonary veno-occlusive disease secondary to lung cancer
}

\author{
N B N Ibrahim, H Burnley, K A Gaber, L J Ormerod, B Addis, F Capron, C R McGavin
}

J Clin Pathol 2005;58:434-436. doi: 10.1136/icp.2004.020735

This report describes two cases of segmental pulmonary vein occlusion secondary to lung malignancy in which lung biopsies showed histological features of veno-occlusive disease. These are the first cases to be reported in the literature in which such lung parenchymal histological changes are described in association with lung malignancy.

n this article, we document two cases of segmental venoocclusive disease associated with squamous cell carcinoma of the lung and pulmonary vein leiomyosarcoma. Although invasion of pulmonary veins by lung carcinoma is not uncommon, ${ }^{12}$ being described in $6 \%$ of tumours, ${ }^{1}$ and leiomyosarcoma involving pulmonary veins has been reported previously, ${ }^{3}$ these are the first cases in which lung parenchymal changes reminiscent of veno-occlusive disease secondary to underlying lung malignancy have been described.

\section{CASE REPORTS \\ Case 1}

A 42 year old woman presented with dyspnoea and a dry cough. She was a lifelong non-smoker, previously well, with no history of allergen exposure, chest disease, or occupational risk factors. She was hypoxic, with a normocytic anaemia, hypoproteinaemia, and a raised erythrocyte sedimentation rate. An autoimmune profile was negative. Respiratory function tests showed a mild restrictive pattern. A chest $x$ ray showed a diffuse interstitial process, with no evidence of pulmonary oedema. A computed tomography (CT) scan (fig 1) showed some lymphadenopathy, pronounced hypovascularity of the right lung, and normal vascularity of the left lung. There was no clinical or radiological evidence of pulmonary hypertension. A transbronchial biopsy failed to yield a diagnosis, so a video assisted thoracoscopic biopsy and mediastinoscopy was attempted, but this was unsuccessful because of dense adhesions in the right pleural cavity. Therefore, a right thoracotomy was performed and a biopsy was taken from the right lung. The right lower lobe was found to be consolidated and densely adherent to the diaphragm. The right lung biopsy showed features of pulmonary veno-occlusive disease (PVOD). After some difficulty weaning her from the ventilator, an echocardiogram revealed a tumour in the left atrium, obstructing the right pulmonary veins. She underwent an emergency exenteration of the left atrium, excision of the tumour, and a right pneumonectomy. No biopsy was taken from the left lung because the abnormal changes that were seen on the CT scans were confined to the right lung. The right lung had a pale surface with a fibrinous haemorrhagic exudate over the lateral aspect. The left atrial tumour was multilobulated and measured $60 \times 50 \times 40 \mathrm{~mm}$, with a partially smooth surface. Histologically, the lung showed patchy but widespread

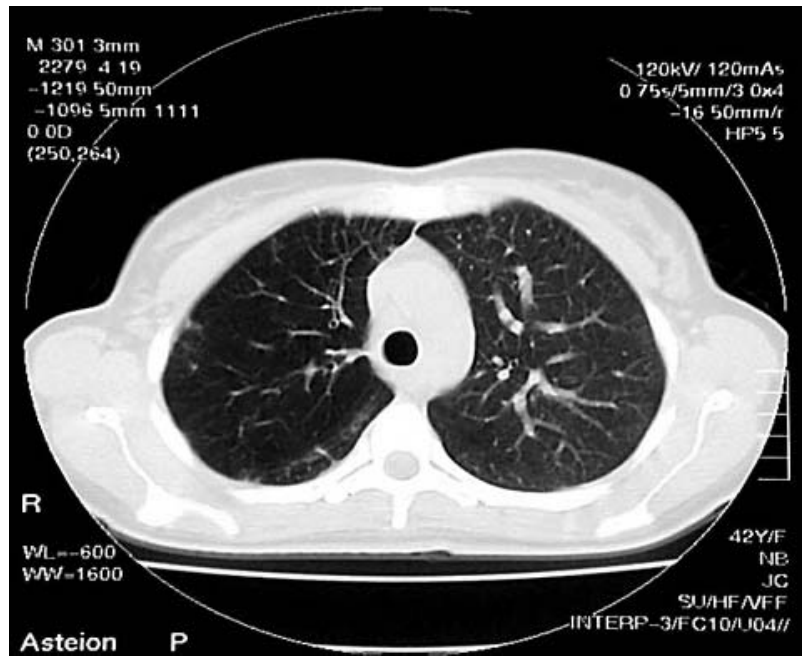

Figure 1 Computed tomography scan showing pronounced hypovascularity of the right lung and normal vascularity of the left lung.

interstitial fibrosis, with features of veno-occlusive disease There was oedema of the pulmonary septa with "arterialisation" of veins, which showed thickened walls and intimal proliferation, and the arteries also showed pronounced intimal thickening, medial hypertrophy, and virtual occlusion in some places (fig 2). No plexiform arterial lesions were seen, and there was no evidence of thromboembolic disease. The intra-atrial part of the tumour, which extended from the right pulmonary veins, was attached to the endothelial surface of the atrium, but did not infiltrate its wall. There was a variable growth pattern, with fascicles of pleomorphic blunt ended spindle cells with morphological and immunohistochemical features of leiomyosarcoma (positive staining for MyoDl and smooth muscle actin, in addition to focal positivity for desmin). The subcarinal lymph nodes received showed no evidence of malignancy.

\section{Case 2}

A 52 year old woman with a 40 year history of smoking presented in October 2000, with a three week history of haemoptysis. Her past medical history included hysterectomy and peripheral vascular disease. She had finger clubbing, but otherwise her physical examination was normal. A chest radiograph showed left upper lobe consolidation without loss of volume. The right lung appeared normal. Lung cancer was suspected and a CT scan revealed left upper lobe consolidation, with a $6 \mathrm{~cm}$ tumour mass and pleural effusion, suggestive of inoperable lung cancer. There was no clinical or radiological evidence of pulmonary hypertension.

Abbreviations: CT, computed tomography; PVOD, pulmonary venoocclusive disease 


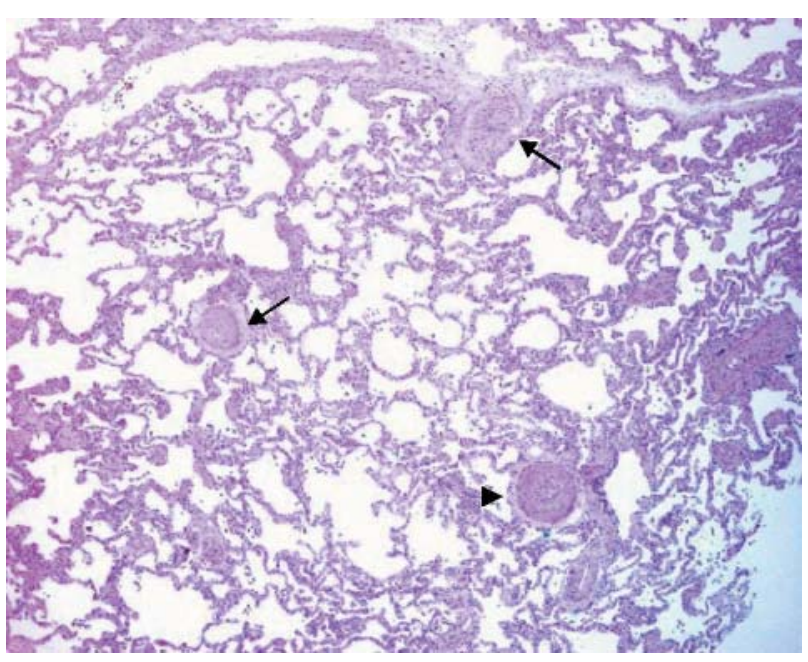

Figure 2 Pulmonary veins (arrows) showing prominent intimal fibrosis. Note also the intimal thickening of the pulmonary artery (arrow head) adjacent to a bronchiole. Haematoxylin and eosin staining.

Fibreoptic bronchoscopy showed blood in the orifice of the lingula. Bronchial washing and CT guided tissue biopsy were negative for malignancy. In January 2001, she underwent video assisted thoracoscopic biopsy. Histologically, the lung showed features of veno-occlusive disease, with widespread accumulation of pigment laden macrophages within numerous alveoli (fig 3A). An iron stain confirmed haemosiderosis (fig 3B). Some of the pulmonary veins showed intimal and medial thickening, associated with narrowing of their lumina, and many of the lymphatics were dilated and contained haemosiderin laden macrophages. An occasional pulmonary artery showed some intimal and medial thickening. No plexiform arterial lesions were seen. There was no evidence of malignancy. Her symptoms persisted and in June 2001 she underwent a rigid bronchoscopy and open lung biopsy. Biopsies from the lingula, which was grossly abnormal, again showed features of veno-occlusive disease, with no evidence of malignancy. She was closely followed up.

In May 2002 she developed headache, right shoulder weakness, and splinter haemorrhages. A transoesophageal echocardiogram revealed a mass in the left atrium, attached to and obstructing the blood flow in the superior pulmonary veins. Left atrial myxoma with systemic embolisation or bacterial endocarditis was suspected. She was treated with intravenous antibiotics. A further CT scan (fig 4) showed a left upper lobe tumour extending directly into the left atrial cavity, close to the left superior pulmonary vein, which was consistent with lung cancer. A repeat fibreoptic bronchoscopy showed a tumour visible at the lingual orifice and a biopsy was taken. Histology showed squamous cell carcinoma. She

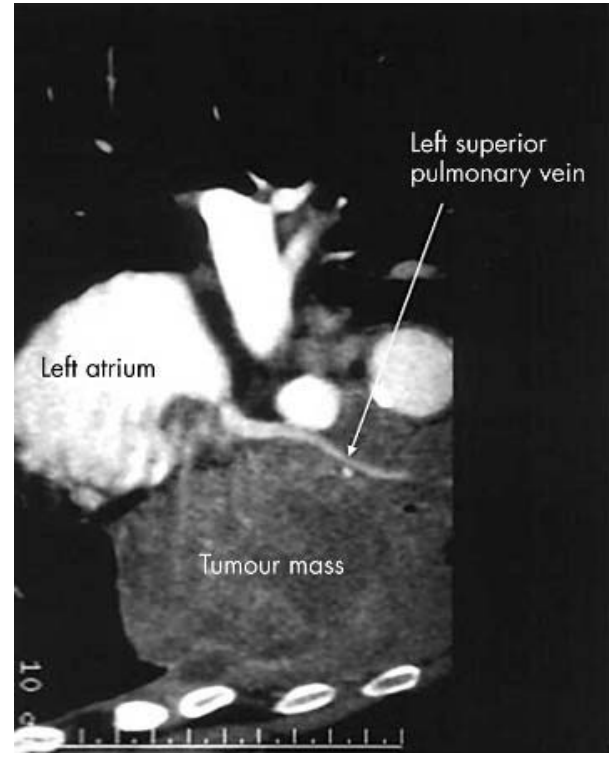

Figure 4 Computed tomography scan showing a left upper lobe tumour invading through the left atrial wall (and obstructing the superior pulmonary veins).

received palliative chemotherapy and died in December 2002. No postmortem examination was carried out.

\section{DISCUSSION}

PVOD is a rare cause of primary pulmonary hypertension characterised by occlusion of intrapulmonary small veins and venules by intimal fibrosis. In most cases, the cause of PVOD is unknown, although the condition has been described in patients receiving chemotherapy, radiotherapy, bone marrow and renal transplantation, and in patients with systemic sclerosis, lower respiratory tract infections, particularly viral infections, and in association with human immunodeficiency virus infection..$^{5-8}$

"The lung parenchymal changes of pulmonary venoocclusive disease in these two cases were caused by invasion of the pulmonary veins by lung cancer"

Histologically, the pulmonary veins and venules show obstructive intimal fibrosis, which is initially loose and oedematous. Often, there is recanalisation with septa formation. Arterialisation of veins may be prominent and the tunica media may show hypertrophy. Recent thrombi are often seen. Dilatation of accompanying lymphatics is common. The pulmonary arteries may show mild to moderate arterial hypertensive changes, with intimal fibrotic

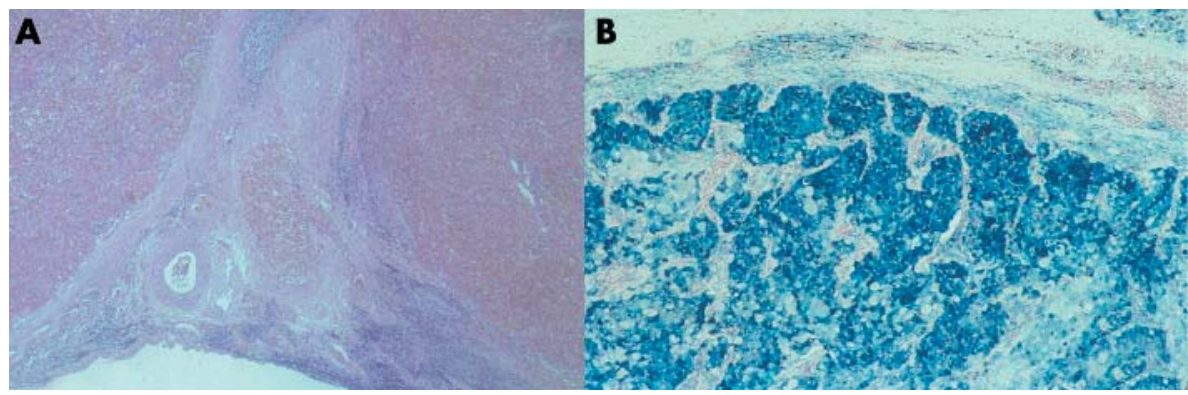

Figure 3 (A) Extensive intra-alveolar haemorrhage with accumulation of pigment laden macrophages. Note the vascular changes. Haematoxylin and eosin stain. (B) Perl's stain for iron confirming extensive pulmonary haemosiderosis. 


\section{Take home messages}

- We report two cases of segmental veno-occlusive disease secondary to lung malignancy

- The finding of histological features of pulmonary venoocclusive disease in lung biopsies coupled with evidence of obstruction of the superior pulmonary vein on computed tomography scans should be regarded as highly suggestive of intrapericardial extension of lung malignancy

changes, often with recent thrombi. The lung parenchyma shows prominent haemosiderosis, focal congestion, and interstitial fibrosis, with fibrous thickening of interlobular septa, which may contain sclerotic veins and dilated lymphatics. ${ }^{9}$ Haemosiderosis, as seen in our second case, is common, and may be associated with iron and calcium encrustation of the wall of the blood vessels, which may elicit a foreign body giant cell reaction. The differential diagnosis of PVOD includes narrowing or obstruction of major pulmonary veins, idiopathic pulmonary haemosiderosis, and primary pulmonary hypertension with other causes. ${ }^{10}$

Pulmonary vein occlusion occurs in $6 \%$ of patients with primary lung cancer, ${ }^{1}$ but parenchymal lung changes reminiscent of PVOD have not been described previously in these patients. In both our cases, there was no clinical or radiological evidence of pulmonary hypertension and the disease process was confined to one lung. We conclude that the lung parenchymal changes of PVOD in these two cases were caused by invasion of the pulmonary veins by lung cancer.

Although surgery was carried out in our first patient, surgical resection in the second patient was not appropriate because of the presence of a pleural effusion on CT scan (stage IV disease). Furthermore, radiotherapy was thought to be hazardous because of the risk of atrial perforation and massive bleeding as a result of involvement of the atrial wall by tumour, which clinically raised the possibility of bacterial endocarditis or atrial myxoma.

\section{ACKNOWLEDGEMENTS}

We would like to thank Dr C S F McCormick, Dr N J Robertson, Dr C B A Lyons, and Dr M E F Smith, Department of Cellular Pathology, Derriford Hospital, Plymouth, UK, for their generous assistance with the second case in this report.

\section{Authors' affiliations}

N B N Ibrahim, Department of Histopathology, Frenchay Hospital, Bristol BS16 1LE, UK

H Burnley, B Addis, Department of Cellular Pathology, Southampton General Hospital, Southampton SO16 6YD, UK

F Capron, Service d'anatomie Pathologique 1, Hôpital de la Pitié, 83, Bd de l'Hôpital, 75651 Paris Cedex 13, France

K A Gaber, L J Ormerod, C R McGavin, Department of Respiratory Medicine, Derriford Hospital, Plymouth PL6 8DH, UK

Correspondence to: Dr N B N Ibrahim, Department of Histopathology, Frenchay Hospital, Bristol BS16 1LE, UK; nassif.ibrahim@dial.pipex.com

Accepted for publication 21 July 2004

\section{REFERENCES}

1 Choe $\mathrm{DH}$, Lee $\mathrm{JH}$, Lee $\mathrm{BH}$, et al. Obliteration of the pulmonary vein in lung cancer: significance in assessing local extent with CT. J Comput Assist Tomogr 1998;22:587-91.

2 Takahashi K, Furuse $\mathrm{M}$, Hanaoka $\mathrm{H}$, et al. Pulmonary vein and left atrial invasion by lung cancer: assessment by breath-hold Gadolinium-enhanced three-dimensional MR angiography. J Comput Assist Tomogr 2000;24:557-61.

3 Gonzalez-Campora R, Rubi-Uria J, Mora-Marin J, et al. Pulmonary vein myxoid leimyosarcoma. Pathol Res Pract 1989; 185:900-6.

4 Gyhra AS, Santander CK, Alarcon EC, et al. Leiomyosarcoma of the pulmonary veins with extension to the left atrium. Ann Thorac Surg 1996:61:1840-1.

5 Knight BK, Rose AG. Pulmonary veno-occlusive disease after chemotherapy. Thorax 1985;40:874.

6 Capewell SJ, Wright AJ, Ellis DA. Pulmonary veno-occlusive disease in association with Hodgkin's disease. Thorax 1984;39:554.

7 Ruchelli ED, Nojadera G, Rutstein RM, et al. Pulmonary veno-occlusive disease. Another vascular disorder associated with human immunodeficiency virus infection? Arch Pathol Lab Med 1994; 118:664.

8 Corrin B. Pulmonary veno-occlusive disease. In: Pathology of the lungs. Edinburgh: Churchill Livingstone, 2000:384-6.

9 Travis WD, Colby TV, Koss MN, et al. Non-neoplastic disorders of the lower respiratory tract. In: Atlas of nontumour pathology. 1st Series, Fascicle 2. Washington DC: AFIP and ARP, 2002:779-81.

10 Katzenstein A-L. Pulmonary hypertension and other vascular disorders. In: Katzenstein and Askin's surgical pathology of non-neoplastic lung disease, 3rd ed. Philadelphia: Saunders, 1997:343-6. 